\title{
Indicadores tecnológicos e organizacionais do Customer Relationship Management (CRM): relação entre firma desenvolvedora, firma usuária e preceitos teóricos
}

Flávio Régio Brambilla
Bacharel em Administração e Especialista em Gestão Organizacional pela ULBRA; Mestre em Administração e Negócios: Marketing e Especialista em Recursos Humanos pela PUCRS; Especialista em Educação a Distância e em Gestão Educacional pelo SENAC; Doutorando em Administração pela UNISINOS; Professor Titular do curso de Administração (Graduação e Pós) da Universidade Luterana do Brasil, ULBRA de Gravataí

\section{Cláudio Hoffmann Sampaio}

\section{Professor Doutor da PUC-RS do} Programa de Mestrado em Administração e Negócios.

Marcelo Gattermann Perin

Professor Doutor da PUC-RS do

Programa de Mestrado em Administração e Negócios.

O Customer Relationship Management (CRM) é um instrumento que viabiliza identificar e atender às necessidades dos clientes. Este artigo verifica as dimensões tecnológica e organizacional do CRM. O estudo envolve uma empresa desenvolvedora de CRM (HP) e uma usuária (Rede Globo). Os resultados indicam que todas as variáveis das dimensões propostas se mostraram pertinentes ao estudo desenvolvido, exceto os sistemas de call center, que inexistem neste caso. A metodologia adotada foi o estudo de caso com triangulação de dados. 
Palavras-chave: Gerenciamento dos relacionamentos com clientes; Tecnologia; Informação.

\title{
Technological and organizational indicators of Customer Relationship Management (CRM): relationship between developer firm, client firm and academic theory
}

\begin{abstract}
Customer Relationship Management (CRM) is an instrument that allows to identify and assist the customers in their needs. This article verifies the technological and organizacional dimensions of CRM. The study involves a company that builds CRM (HP) and a user firm (Rede Globo). The results indicate that all variables of the proposed dimensions were pertinent to the study carried out, except for the call center system, that are inexistent in this case. The adopted methodology was case study with data triangulation.
\end{abstract}

Keywords: Customer relationship management - CRM; Technology; Information.

Recebido em 03.12.2007 Aceito em 18.06.2008

\section{Introdução}

O atendimento e a verificação das necessidades latentes dos clientes constituem fatores decisivos para a sobrevivência das organizações, independentemente do segmento em que atuam e da natureza de suas operações. As empresas focadas na perspectiva dos clientes abordam a construção de relacionamentos como o diferencial competitivo almejado na atualidade (BERRY, 2002; WINER, 2001a; ROWE e BARNES, 1998).

Entretanto, o estabelecimento de relacionamentos no cenário competitivo atual, caracterizado por turbulência e rápidas mudanças, requer um monitoramento mais próximo e informações mais freqüentes e precisas, impulsionando as empresas a tratar as informações de uma forma estratégica e fundamental, uma vez que possibilita identificar as mudanças na arena competitiva, aprimorando respostas, reposicionamentos e decisões.

Dessa forma, as transformações no ambiente de negócio impelem que sejam conhecidos os fatores de mudanças e as novas necessidades de gestão e dos clientes, aumentando o valor das informações à tomada de 
decisão (MAFRA PEREIRA e BARBOSA, 2008). A informação passa, então, a constituir-se em capital tão vital como outros ativos organizacionais, como um insumo básico para o desenvolvimento das diversas atividades da empresa (VALENTIM et. al., 2008), assumindo papel primordial na atividade gerencial estratégica e de relacionamento (JAMIL e NEVES, 2000).

Portanto, a capacidade para tomar decisões estratégicas com eficiência está estreitamente relacionada com sistemas estruturados capazes de gerenciar as informações dentro das empresas, promovendo fluxos eficientes que aprimorem a qualidade das decisões, disponibilizando à administração informações necessárias e em tempo, local e formato adequados (GOULART, 2007).

Neste contexto, ressalta-se a importância do Customer Relationship Management (CRM) como uma estratégia de gestão que utiliza informações do mercado e da empresa, e viabiliza a identificação e o atendimento das necessidades dos clientes. O CRM é definido como uma abordagem gerencial que propicia às organizações a identificação, a atração e o aumento da retenção dos clientes, e que proporciona uma maior rentabilidade para a empresa justamente desenvolvendo ações para a identificação e para o aumento das transações com os clientes de maior valor. As ações de CRM são dimensionadas levando em consideração o foco do relacionamento com clientes, e residem no entendimento de seu comportamento de compra, de pagamento, bem como do tipo de consumo que efetuam (SHETH e PARVATIYAR, 2002). Lin e Su (2003, p.716) definem CRM como sendo "a chave da competição estratégica necessária para manter o foco nas necessidades dos clientes e para uma abordagem face-a-face com o cliente ao longo da organização".

Para que a empresa possa usufruir dos benefícios do CRM como abordagem gerencial, a definição de indicadores tecnológicos e gerenciais constitui fator crítico. Pedron (2003) afirma que a definição dos indicadores de sucesso em CRM é uma das atribuições mais difíceis do ponto de vista estratégico. Ainda, Ceretta e Quadros (2003) destacam que é de grande importância e dificuldade o desenvolvimento de indicadores de desempenho que apresentem consistência. Neste sentido, este trabalho objetiva ampliar a compreensão dos atributos necessários para a efetiva prática do CRM. Para uma maior abrangência organizacional e maior capacidade de informações sobre o comportamento dos clientes, neste trabalho foram investigadas as dimensões tecnológica e organizacional do CRM. Para tanto, foi realizado um estudo de caso envolvendo duas empresas: uma desenvolvedora de CRM (HP) e uma usuária (Rede Globo).

O artigo encontra-se estruturado da seguinte forma: após esta introdução, uma revisão teórica sobre o CRM, englobando o foco de relacionamento e a necessidade de informações, além das dimensões tecnológica e organizacional do CRM; a seguir, o método utilizado na pesquisa; na seqüência, os resultados na forma de congruências obtidas no estudo; por fim, algumas considerações finais. 


\section{CRM: foco de relacionamento e necessidade de informação}

A filosofia das empresas tem sofrido um redirecionamento de foco, de uma ênfase em aquisição de clientes para outra na gestão de relacionamentos. Para Ragins e Greco (2003), o CRM tem um papel decisivo nessa nova postura empresarial, mapeando e delineando as percepções dos clientes sobre a organização e seus produtos, através da identificação destes clientes; criando o conhecimento do consumidor e construindo relações com os mesmos. Para que isto ocorra, existe a necessidade de a empresa possuir quantidade de informações adequadas para as pessoas certas e no tempo certo.

Miranda (2006) define necessidade de informação como um estado no qual se percebe a insuficiência ou inadequação dos conhecimentos necessários para solucionar os problemas da empresa. Para Le Coadic (2003), a necessidade de informação é evolutiva e extensiva, pois muda com o tempo; é dinâmica, e cada nova informação acaba gerando novas necessidades. Em relação às necessidades e uso da informação, Dervin e Nilan (1986) ressaltam que o usuário da informação deve ser o foco central da análise, e não os sistemas que ele utiliza - as necessidades dos usuários devem direcionar os sistemas de informação, isto é, os serviços de informação devem ser ajustados às necessidades específicas dos usuários, e não o contrário.

Depois de a empresa ter atendido à sua necessidade de informação, é possível conhecer os seus clientes, interagir com os mesmos e estreitar relacionamentos. Croteau e $\mathrm{Li}$ (2003) destacam que as informações adequadas são essenciais ao CRM, habilitando a organização a fornecer produtos e serviços específicos para cada cliente, de forma individual, estreitando relações de longo prazo. Segundo Dwyer, Schurr e Oh (1987), as empresas beneficiam-se quando nutrem laços relacionais com os clientes, os quais conduzem para negócios mais seguros e repetidos. Wilson, Daniel e McDonald (2002) destacam a necessidade de as empresas buscarem informações sobre o mercado e possuírem uma ênfase mais balanceada de relacionamento contínuo e mais impactante do que as simples transações individuais e transacionais realizadas sem o conhecimento explícito dos clientes.

A seguir, passa-se a discutir as variáveis que compõem as dimensões tecnológica e organizacional do CRM.

\section{Dimensão tecnológica do CRM}

A dimensão tecnológica do CRM engloba as seguintes variáveis: Tecnologia da Informação (TI), banco de dados, dados dos clientes, Data Warehouse, Data Base Marketing, Data Mining, Call Center e Sales Force Automation (SFA), caracterizadas a seguir. 


\section{Tecnologia da informação (TI)}

A Tecnologia da Informação (TI) consiste no termo que engloba toda a tecnologia utilizada para criar, armazenar, trocar e usar informação em seus diversos formatos. Brown (2001, p.161) afirma que "uma solução de CRM exigirá a adoção de novas tecnologias para alcançar transparência e visibilidade na cadeia de valores dos negócios e entre os negócios e seus clientes". A tecnologia a ser adotada é diretamente relacionada com a estrutura, o negócio e os objetivos da empresa usuária do CRM. Hansotia (2002, p.129) decreve a TI como "o elemento facilitador na execução da estratégia de CRM", chamando a atenção para o fato de que a execução tecnológica do CRM freqüentemente apresenta o desafio da integração de sistemas.

Parvatiyar e Sheth (2001) direcionam o foco para a abordagem de que várias ferramentas de software e outras tecnologias reivindicam soluções para diversos aspectos do CRM e foram recentemente introduzidas para a aplicação comercial. Para Campbell (2003, p.375) "as empresas que adotaram a TI direcionada para a utilização do CRM assim o fizeram em busca de bases de dados necessárias para a avaliação do status e da lucratividade dos seus clientes". A dimensão tecnológica do CRM trabalha com o gerenciamento de quantidades de dados que não podem ser utilizados sem o adequado armazenamento. Os bancos de dados são a próxima variável tecnológica do CRM.

\section{Banco de dados}

O banco de dados é um conjunto de dados adequadamente estruturados, que por sua vez proporcionam a capacidade de utilização eficiente e direcionada para uma gama de aplicações na organização. Segundo Nogueira, Mazzon e Terra (2004, p.13), "uma boa administração de dados é essencial à prática do CRM". Os autores ressaltam que se relacionar com o cliente envolve aprendizado, e para tanto é imprescindível que os bancos de dados utilizados nas práticas de CRM estejam em constante atualização, para que as mudanças de comportamento dos clientes sejam detectadas.

Conforme Dowling (2002), o CRM dirigido por base de dados apresenta melhorias significativas na identificação de clientes lucrativos; e alerta quanto aos não lucrativos, aumentando a eficiência e a efetividade perante os objetivos de marketing. Também aumenta a satisfação do cliente, quando torna possível melhor conhecê-lo e atendê-lo. O uso do banco de dados também responde pelo alinhamento estratégico da TI, com a missão e com os objetivos do negócio da empresa usuária do CRM. Conforme Missi, Alshawi e Irani (2003, p.1608), "a meta da integração de dados é permitir a organização de combinar, agregar e relatar mediante os dados de diferentes fontes", o que permite uma maior capacidade de delinear o padrão comportamental do cliente em foco. 


\section{Dados dos clientes}

O usuário, para desempenhar adequadamente ações de CRM, necessita de dados fidedignos de clientes. Os ganhos das empresas possuidoras de bancos de dados de clientes são relevantes, dentre os quais a acessibilidade aos clientes, a mensuração das transações efetuadas com estes, a flexibilidade em atender de forma individualizada cada tipo de cliente, e a capacidade de contabilização do valor de cada cliente (ou categorias de cliente).

Para Nogueira, Mazzon e Terra (2004), além dos aspectos de funcionamento adequado do sistema, como a eliminação de redundância e duplicidades, também é importante a perspectiva deste em termos de segurança, de garantia de integridade e de privacidade dos dados de clientes, o que é fundamental para o sucesso da aplicação de CRM. De acordo com McKim (2002), os dados ajudam na descoberta do que é necessário para uma comunicação efetiva com o cliente.

Hansotia (2002, p.121) salienta que o "CRM é essencialmente um esforço intensivo com dados de clientes". O autor também afirma que no centro do CRM está a habilidade organizacional de nivelar dados de clientes de forma criativa, efetiva e eficiente para desenvolver estratégias focadas em clientes. Missi, Alshawi e Irani (2003, p.1607) dizem que "a essência do sistema de CRM implica em entender, controlar e aperfeiçoar os negócios e o gerenciamento dos dados". Campbell (2003) coloca que os dados de clientes devem ser transformados pelos gestores em informações de clientes, e estas devem então ser integradas na totalidade. Shoemaker (2001) considera que as interações entre clientes e transações em processo proporcionam uma riqueza de dados e informações que devem ser transformadas em conhecimento de clientes. Os dados de cliente, bem como as demais aplicações tecnológicas de CRM, necessitam de informações confiáveis. Para o fornecimento de tais informações discute-se o data warehouse.

\section{Data warehouse}

O Data Warehouse responde pelo fornecimento das informações confiáveis que suportam o processo da tomada de decisão. Greenberg (2002) menciona tratar-se de um repositório único, depurado, consolidado e consistente, que responde pelo fornecimento de informações confiáveis. Estas informações serão utilizadas como suporte ao processo de tomada das decisões. Nogueira, Mazzon e Terra (2004, p.3) mencionam que Data Warehouse é "o nome genérico para a infraestrutura de armazenamento on-line de dados, utilizada para 0 armazenamento de informações detalhadas sobre os clientes". À medida que agregam informações dispersas, os Data Warehouse possuem um papel fundamental nos modelos de negócio que priorizam a informação como um bem estratégico (JAMIL e NEVES, 2000). Um aspecto muito importante, em termos de Data Warehouse, reside na necessidade de que dados sejam 
transformados em informações, que são os elementos fundamentais às práticas de CRM. O Data Warehouse tem sua razão de existir, segundo Angelo e Giangrande (1999), em virtude da necessidade de integração dos dados corporativos em um único local, para que estes fiquem então acessíveis a todos os usuários envolvidos nos níveis de decisão da empresa. De acordo com Swift (2001), servem para armazenar informações históricas em detalhes e centradas no cliente, desta forma permitindo que as empresas se tornem ágeis e responsivas no mercado, podendo então tomar decisões sólidas de marketing. Srivastava et al. (2002) dizem que a construção de Data Warehouse é um passo importante para o início do CRM analítico. Para uma adequada gestão dos relacionamentos entre empresa e cliente, a empresa necessita de dados em tempo e adequados para as ações. Estes dados precisam demonstrar o perfil dos clientes.

\section{Database marketing}

Dados sobre o perfil dos clientes atuais (registros das vendas realizadas) e potenciais (prospects) são aspectos primordiais para o entendimento do funcionamento do Database Marketing. Consiste em um gerenciamento dinâmico de uma base de dados atualizada com as informações relevantes dos clientes, sejam estes os atuais ou potenciais, tendo como principal vantagem a atualização constante de um grande número de informações dos clientes (PEDRON, 2003). Para Hughes (1998), Database Marketing deve ser configurado com os dados que demonstram o perfil dos clientes atuais e potenciais, apresentando seu histórico de compra, para que o sistema atenda ao propósito de agregar as informações sobre cada cliente, e seja gerenciado o relacionamento personalizado. Para a possibilidade de ser realizada uma completa solução de CRM, segundo Winer (2001a), é necessária a construção de uma base de dados contendo as informações mais completas possíveis de clientes. Fletcher (2003) relata que as empresas estão aglomerando uma cada vez maior quantidade de informações pessoais dos clientes nas transações, especialmente online, e utilizando-as para o aprimoramento das vendas e da efetividade dos serviços prestados.

\section{Data mining}

Conforme Srivastava et al. (2002), Data Mining, ou mineração de dados, responde pela análise das informações em um banco de dados, pelo processo de extração e cruzamento de informações relevantes e daquelas características que representem o modelo comportamental do cliente. O seu foco primário é voltado para a busca de conhecimento, anteriormente não existente ou disponível, utilizado para predizer o futuro e automatizar a análise de uma grande quantidade de conjuntos de dados. Bretzke (2000) descreve a utilização de Data Mining como uma ferramenta utilizada na busca de clientes mais rentáveis, ou segmentos de 
clientes mais significativos para a empresa. Paas e Kuijlen (2001, p.57) reportam que Data Mining é "particularmente crucial para transformar dados transacionais armazenados em insights sobre as necessidades do cliente".

\section{Call center - centros de interação com clientes (CICs)}

Um Call Center é definido como um sistema amplo e de contato dinâmico de relacionamento com os clientes, centralizando suas informações. É também definido como um meio de interação com os clientes, operacionalizado com base na filosofia do CRM (BRETZKE, 2000). Salazar (2002) classifica Call Center como o nome dado para as centrais de atendimento empresarial, nas quais são utilizadas técnicas de marketing e de telemarketing integradas com as ferramentas de telecomunicações. O sistema de Call Center é uma ferramenta tecnológica adequada para a utilização em um canal de relacionamento. Salazar (2002, p.60) afirma que "esse canal de relacionamento deve servir, também, como um suporte a vendas e administração com a intenção de manter o cliente sempre próximo e conhecedor das práticas da empresa". Shoemaker (2001, p.180) diz que "o avanço tecnológico está aumentando as capacidades dos call centers", e que "o apropriado uso destas ferramentas é crucial para o desenvolvimento de lealdade positiva nos relacionamentos com os clientes". A evolução está resultando na transição dos Call Centers tradicionais para a sua mais evoluída faceta - os chamados Centros de Interação com Clientes, ou CICs.

\section{Sales force automation (SFA)}

A Sales Force Automation (SFA) refere-se ao software para automatização de força de vendas. Shoemaker (2001) afirma que a maioria dos provedores de software de CRM tem suas raízes em SFA. Speier e Venkatesh (2002, p.98) destacam que "as tecnologias de automação da força de vendas (SFA) estão sendo cada vez mais utilizadas no suporte das estratégias de CRM", e que as ferramentas de SFA são freqüentemente utilizadas para facilitar os processos de CRM como parte da filosofia do marketing de relacionamento.

Algumas das características organizacionais podem ter uma influência significante em uma adoção tecnológica de sucesso, também impactando na aceitação da SFA. É fato que o pessoal de vendas reage de forma razoavelmente positiva em relação às ferramentas de SFA, imediatamente após o treinamento. Porém, esta resposta inicial se torna negativa após a utilização das ferramentas por um tempo maior (SPEIER e VENKATESH, 2002). Segundo os autores, o pessoal de vendas deve estar envolvido na implementação do processo e, depois, ser adequadamente treinado para manter-se efetivo em sua utilização, entendendo a importância e a ajuda que o SFA pode trazer ao atendimento do cliente de 
forma personalizada e com mais qualidade, permitindo o acesso a toda história da relação da empresa com o cliente.

\section{Dimensão organizacional do CRM}

A dimensão organizacional do CRM engloba planejamento e monitoramento do projeto de CRM, recursos humanos, mudança na filosofia empresarial e poder, tratados a seguir.

\section{Planejamento e monitoramento do projeto de CRM}

O projeto de CRM é, por definição, um projeto corporativo. GrabnerKraeuter e Moedritscher (2002, p.3) salientam que "CRM é uma estratégia, não uma tecnologia". Embora o CRM utilize tecnologia e equipamentos, ainda assim a essência de sua aplicação e efetividade emerge da filosofia, e não das ferramentas utilizadas no processo; necessitando, portanto, de planejamento adequado.

Abordando a amplitude da estratégia de negócio, Norris e Hurley (2001) dizem que o CRM envolve a combinação entre pessoas, processos e sistemas. Tem-se então que esta tríade de elementos no CRM é direcionada para o melhor e mais adequado atendimento do cliente. Para Missi, Alshawi e Irani (2003), o desenvolvimento de uma estratégia de negócio convergente com o CRM ajuda as empresas no reconhecimento e na manutenção de clientes de valor, alocando então recursos para aumentar o valor de cliente.

Em relação ao monitoramento do projeto de CRM, a importância da ação se dá em virtude da necessidade de elaboração e criação de um projeto que seja realístico e pragmático, em que estas ações sejam realizadas periodicamente, conforme os objetivos estratégicos e econômicos previstos no início do projeto; onde será efetivada a comparação entre os objetivos e metas definidas em relação aos resultados efetivamente alcançados. Outro aspecto de grande importância destacado por Newell (2000) é a necessidade de participação de todos no projeto estrutural de CRM. Angelo e Giangrande (1999) afirmam que, no projeto de implantação do CRM, todos os funcionários da empresa, em menor ou maior grau de envolvimento no projeto, devem ter a consciência da importância de sua contribuição. Hansotia (2002, p.132) alerta que o "CRM depende de cuidadoso planejamento e prontidão organizacional", o que inclui a monitoração. Portanto, toda a organização tem de estar preocupada com a coleta e com o armazenamento dos dados do cliente, bem como com o objetivo de conhecer melhor cada um deles. Esta tarefa requer uma adequada estrutura de Recursos Humanos.

\section{Recursos humanos}

Os Recursos Humanos aparecem como uma variável fundamental, que requer adaptação para a aplicação efetiva das filosofias e ações de 
CRM. Em relação aos envolvidos nos processos organizacionais, conforme Souza (2005), a busca pelo desempenho nas atividades do trabalho, seja ele qual for, é primordial para o sucesso das organizações. È constatado que trabalhadores satisfeitos, motivados e integrados ao processo de trabalho produzem resultados mais expressivos e de excelência para organização. Segundo Pedron (2003), é preciso que o colaborador organizacional tenha a consciência de sua importância com relação ao papel que cumpre na empresa. Sendo assim, sua participação deve ser incentivada, para que venha a dar sugestões sobre as questões que requerem solução. Para Parvatiyar e Sheth (2001), várias decisões de recursos humanos são também importantes para a criação da organização certa e com clima para a administração da gestão de relacionamento com o cliente.

\section{Mudança na filosofia empresarial}

Com relação às mudanças necessárias na filosofia empresarial para a adoção do CRM, tem-se no primeiro momento a reavaliação dos objetivos, valores e missão da empresa, ou seja, elementos estruturais. Mais do que isso, mudanças na própria cultura da empresa. As mudanças na cultura empresarial decorrem da mudança na postura dos agentes organizacionais em geral, em especial das lideranças. Quando se trata de mudança nas filosofias empresariais, também emerge como aspecto importante a reavaliação da estrutura organizacional, para que esta receba a devida adequação aos sistemas e filosofia do CRM. Hansotia (2002) destaca que o CRM coloca o cliente no cerne da organização. Desta maneira, todos os processos da empresa são projetados para o ponto de vista do cliente, para gerar maior lealdade e aumentar o valor do cliente no longo prazo. Hansotia (2002) argumenta que o CRM não obterá sucesso se for apenas o sonho da média e da alta gerência ou da cúpula organizacional. É preciso o total compromisso na organização, não apenas do administrador geral, mas sim de todos os demais gestores e colaboradores.

\section{Poder}

No contexto do CRM, é preciso a concessão de autonomia suficiente aos colaboradores da empresa para a realização das suas tarefas diárias. A antiga visão da delegação de ordens deve ser substituída pelo empowerment. Ferreira e Sganzerlla (2000) afirmam que o empowerment é o mesmo que difundir ou delegar poder, o que aparece como um dos mais importantes aspectos de mudanças através do processo de implantação da gestão de relacionamento com o cliente.

Além da delegação de poder, Bretzke (2000) diz que um dos aspectos mais importantes e decisivos para que o projeto atinja o sucesso desejado é a sua condução por uma liderança com capacidade de resolução dos conflitos, e que consiga então a garantia e a cooperação na 
relação entre as equipes envolvidas no projeto. Os líderes que queiram atingir níveis de excelência, na visão de Souza (2005), devem entender a diferença individual de cada um dos elementos participantes do processo de implantação do sistema de CRM, e, desta forma, agir segundo os níveis de competências dos liderados, assumindo assim papéis diferenciados.

Em síntese, o ambiente competitivo e a mudança de foco empresarial para relações contínuas impeliram um status superior ao CRM e, por conseguinte, às informações adequadas como recurso empresarial estratégico. Após a empresa definir sua necessidade de informações, as ações de CRM podem ser implementadas, habilitando a organização a fornecer produtos e serviços específicos a cada cliente. Essas ações de CRM englobam as dimensões tecnológica (tecnologia da informação, banco de dados, dados dos clientes, data warehouse, database marketing, call center, sales force administration) e organizacional (planejamento e monitoramento do projeto, recursos humanos, filosofia empresarial, poder) que são estruturas paralelas e complementares, necessitando-se de alinhamento e adequação entre elas e com a estratégia empresarial adotada.

\section{Método}

Dado o objetivo proposto, optou-se pela realização de uma pesquisa qualitativa, de natureza exploratória. Para tanto, buscou-se uma forma compreensiva e interpretativa dos fenômenos, de maneira a proporcionar uma visão geral com vistas à compreensão dos atributos necessários para a efetiva prática do CRM, tendo como base os indicadores tecnológicos e organizacionais que permeiam a sua aplicação. Tal definição se fundou nas afirmações de Patton (1986), para o qual os estudos de natureza qualitativa contribuem com uma visão do relacionamento das variáveis a partir de um determinado contexto (visão holística), possibilitando que se parta de questões mais amplas, deixando a natureza mais específica das variáveis ou do fenômeno emergir durante o processo (abordagem indutiva); com a ausência de intervenção do pesquisador, no caso estudado (investigação naturalística).

A estratégia de pesquisa escolhida para o desenvolvimento do trabalho foi a de Estudo de Casos, que busca aprofundar o estudo sobre um determinado objeto, a fim de adquirir conhecimentos mais aprofundados sobre o mesmo (GIL, 1999). O Estudo de Caso permite, segundo Bryman (1992), obter insights e encontrar evidências empíricas que auxiliem na validação de teorias.

Em função dos objetivos da pesquisa, o estudo considerou um caso de ambiente organizacional que apresentasse destaque nas variáveis em estudo, especialmente a efetiva aplicação de programa de CRM; envolvendo uma empresa prestadora de serviços de CRM e uma de suas empresas clientes mais expressivas na aplicação deste tipo de programa. Mais especificamente, selecionou-se a unidade da empresa Hewlett Packard (HP) do Parque Tecnológico (TECNOPUC) da Pontifícia 
Universidade Católica do Rio Grande do Sul (PUCRS), além de uma de suas principais empresas clientes, qual seja a Rede Globo de Televisão. Tal seleção teve por base a maior evidência dos construtos envolvidos no fenômeno em estudo. A unidade de análise, perante o caso escolhido, foi o processo de aplicação de um programa de CRM pela empresa prestadora em sua empresa cliente.

Para a realização deste estudo, foram realizadas cinco etapas básicas, as quais permitiram aos pesquisadores determinar e acompanhar da melhor forma o andamento dos trabalhos. Esse delineamento contribuiu para uma melhor visualização do escopo dos procedimentos metodológicos adotados, garantindo, assim, maior validade e confiabilidade dos construtos (GIL, 1999; YIN, 2005).

A primeira etapa do estudo envolveu a revisão da bibliografia especializada, com o objetivo de se identificar os construtos e as relações iniciais (categorias a priori) da pesquisa. O desenvolvimento prévio da teoria, enfocando as dimensões tecnológica e organizacional do CRM, facilitou a fase de coleta de dados nas empresas selecionadas, direcionando quais dados deveriam ser coletados, bem como sua análise posterior (BARDIN, 1977; YIN, 2005). Para tanto, foi fundamental a definição dos construtos e relações da pesquisa a partir da fundamentação teórica, definindo o roteiro de análise dos casos (segunda etapa). Esta etapa foi subsidiada por reuniões realizadas com pesquisadores do Mestrado em Administração e Negócios (MAN) da PUCRS. Essencialmente, o roteiro de análise foi formado com as dimensões e subdimensões supracitadas nos itens 3 e 4.

Já na terceira etapa, o roteiro de análise foi validado por especialistas com experiência prática profissional e acadêmica. Para tanto, foram apresentados o tema e os objetivos de pesquisa para que os especialistas consultados pudessem efetuar críticas, dar sugestões, apontar novas dimensões e realizar, dessa forma, a sua validação. Na seqüência, foi realizada uma adequação da lista de dimensões da pesquisa mediante as novas proposições dos especialistas, objetivando um refinamento do roteiro de análise (HOPPEN, LAPOINTE e MOREAU, 1996). Registra-se que durante a pesquisa não foi excluída a possibilidade de novas dimensões emergirem na investigação dos dados.

A coleta de dados ocorreu na quarta etapa. As formas de coleta de dados aplicadas no estudo de caso envolveram um processo de triangulação de métodos para a compreensão e contraposição dos dados levantados (GIL, 1999; YIN, 2005). Foram elas: (a) entrevistas em profundidade com três gestores do projeto de CRM da HP e com o gestor do processo de CRM na empresa Globo, além de dois especialistas sobre o tema; e (b) análises de documentos internos e externos das empresas focadas, especialmente material promocional, manuais de procedimentos e contratos entre as empresas, relativos ao programa de CRM. Os registros das entrevistas foram realizados mediante gravações e posteriores transcrições, sendo os entrevistados definidos por julgamento, 
obedecendo ao critério de serem pessoas com uma boa visão sobre o processo de CRM da empresa.

Para aumentar a validade de uma interpretação sobre uma afirmação ou dado coletado a partir de uma entrevista, a análise de documentos ocorreu após a etapa de entrevistas. A partir disso, buscou-se a triangulação das formas de coleta (entrevistas-análise documental) objetivando, além de confirmação do significado, informações adicionais (YIN, 2005).

Após a coleta dos dados, os mesmos foram analisados (quinta etapa) à luz da fundamentação teórica, e organizados a partir das dimensões selecionadas no protocolo da pesquisa. Para haver uma maior sustentação dos dados levantados em relação aos pressupostos do trabalho, foram abordadas as dimensões de pesquisa a partir dos casos relatados na fundamentação teórica, e através de uma interpretação holística; buscando-se identificar provas convergentes com respeito aos fatos e às conclusões para o estudo, sempre se utilizando da triangulação de fontes - entrevistas e documentos (GIL, 1999; YIN, 2005).

Os dados coletados foram analisados mediante a técnica de análise de conteúdo (BARDIN, 1977), com a abordagem temática ou categorial. Os achados foram analisados em função das idéias ou palavras que eles continham (enunciados), sendo estas determinadas a partir do referencial teórico e diretamente relacionadas às dimensões pesquisadas. O conteúdo foi ordenado e integrado em função dos objetivos perseguidos (FREITAS, CUNHA JR., MOSCAROLA, 1996).

Os resultados encontrados em cada empresa foram comparados entre si, buscando-se as convergências e discrepâncias entre os achados. Além disto, todo o conjunto de resultados analisados foi apresentado aos pesquisadores do MAN para uma discussão e aproximação com os resultados por eles encontrados nos ambientes empresariais de seus respectivos países.

O método de pesquisa adotado objetivou, deste modo, estabelecer procedimentos detalhados de coleta, análise e interpretação dos resultados, a fim de garantir uma investigação naturalística, tentando-se minimizar impressões subjetivas do pesquisador e buscando dados a partir de um tratamento qualitativo. A seguir são apresentados os resultados do estudo.

\section{Congruências do estudo}

Os resultados congruentes do estudo foram verificados mediante a comparação entre a teoria apresentada, a situação da empresa desenvolvedora de soluções, e também a análise dos mesmos aspectos no ambiente da empresa cliente e usuária das soluções de CRM. O sistema de CRM da Rede Globo, o chamado SIS.com, é utilizado para a venda de espaço comercial em todo o país. A seguir é apresentado o QUADRO 1 com os resultados referentes à dimensão tecnológica 
QUADRO 1 - Resultados da Dimensão Tecnológica do CRM

\begin{tabular}{|c|c|}
\hline $\begin{array}{l}\text { VARIÁVEIS DA DIMENSÃO } \\
\text { TECNOLÓGICA DO CRM }\end{array}$ & CONGRUÊNCIAS OBTIDAS NO ESTUDO \\
\hline $\begin{array}{l}\text { Tecnologia de Informação } \\
\text { (TI) }\end{array}$ & Indicador congruente na relação HP-Globo-Teoria \\
\hline Banco de Dados & $\begin{array}{l}\text { Indicador parcialmente congruente. A HP não conhece } \\
\text { a plena utilização da variável na relação com o cliente }\end{array}$ \\
\hline Dados dos Clientes & Indicador congruente na relação HP-Globo-Teoria \\
\hline Data Warehouse & Indicador congruente na relação HP-Globo-Teoria \\
\hline Data Base Marketing & $\begin{array}{l}\text { O indicador não é congruente, e, embora viável, não } \\
\text { é uma ferramenta potencializada }\end{array}$ \\
\hline Data Mining & $\begin{array}{l}\text { Indicador congruente, embora a HP não visualize esta } \\
\text { prática na Rede Globo }\end{array}$ \\
\hline Call Center - CICs & $\begin{array}{l}\text { Variável inexistente no contexto tecnológico entre HP } \\
\text { e Globo }\end{array}$ \\
\hline $\begin{array}{l}\text { Sales Force Automation } \\
\text { (SFA) }\end{array}$ & $\begin{array}{l}\text { O indicador é congruente na relação entre as } \\
\text { empresas, mas é contrário ao que a teoria referencia. } \\
\text { As resistências internas e os conflitos relatados pela } \\
\text { teoria não ocorreram no caso HP-Globo }\end{array}$ \\
\hline
\end{tabular}

Fonte: Elaborado pelos autores.

Quanto à Tecnologia de Informação, a congruência é total, conforme pode ser observado no QUADRO 1. A HP desenvolve a solução mediante demanda da empresa cliente Rede Globo, e por isso não incorre em problemas como erros de especificação. A empresa utilitária exerce vendas padronizadas, e verifica o aumento de qualidade nas operações, despontando como líder no mercado de atuação. Possui uma vantagem competitiva, e, em aspectos teóricos, está alinhada com a teoria e com a visão da empresa HP. O contato com o cliente da Rede Globo é maximizado e adequado. Embora remeta à utilização de uma estrutura complexa, permite a realização das práticas de CRM.

O banco de dados é uma variável com um nível relativo de congruência. Apesar de a HP ter o domínio da ferramenta, não possui o conhecimento total das ações da empresa usuária. Com relação aos aspectos técnicos, ocupa o patamar de variável congruente. A ferramenta é utilizada pela TV Globo no desempenho de ações junto aos clientes, e também para ações de marketing, características do CRM. O aspecto não congruente com relação ao banco de dados pode ser suprimido através da apresentação, por parte da Rede Globo, de suas práticas com o banco de dados quando do pedido por melhorias junto à desenvolvedora. Também a empresa HP pode verificar estas práticas para a maximização dos efeitos desta ferramenta, a qual contém toda a memória organizacional referente às operações e clientes.

O uso dos dados de clientes e a compreensão da importância da utilização destes representaram uma variável de congruência total. Um dos aspectos condicionantes para esta congruência é a atualização, a qual 
é praticamente imediata por se tratar de uma ação on-line. O alinhamento adequado destes dados é integrante da solução HP. A Rede Globo utilizaos no contato com os clientes, em ações de CRM. Não foram detectadas duplicidades e outros graves problemas nos dados, apenas uma pequena margem de erro sem impacto na qualidade dos serviços prestados pela Rede Globo de Televisão a seus clientes.

As variáveis data warehouse, data base marketing e data mining, do ponto de vista técnico, estão congruentes. Embora exista certo grau de inconsistência conceitual, no que se refere à viabilidade das ações efetivas no ambiente da empresa usuária, algumas práticas não utilizadas podem ser efetuadas sem grandes modificações e isentas de maiores problemas. Foi detectada a incongruência conceitual, o que também pode ser relatado como incongruência de linguagem. Porém, solucionada a questão nomenclatura, as práticas são viáveis e o alinhamento entre empresas e teoria remetem à situação congruente. A empresa cliente não se utiliza da totalidade do potencial destas ferramentas, mas realiza práticas de CRM adequadas quando levado em conta o cenário de atuação e o tipo de transação exercida com os clientes.

Call center e derivados não foram relevantes para este estudo. Um dos fatores pode ser verificado na natureza das organizações. Trata-se de grandes empresas, detentoras de vantagem competitiva e atuantes no mercado de tecnologia. Outras ferramentas de interação como a SFA, e outros meios de contato, podem justificar a ausência da variável.

$\mathrm{Na}$ relação entre as empresas, e na compreensão mútua da aplicabilidade e de desenvolvimento, a variável SFA mostrou-se congruente. $O$ aspecto que impede considerar-se a totalidade da ferramenta decorre da percepção diversa da prática com a teórica. Do ponto de vista tecnológico, existe a tríade congruência. Já quanto aos efeitos esperados, a teoria enfatiza a possibilidade da geração de conflitos na organização adotante perante a equipe de vendas, o que na prática não ocorreu. Ambas as empresas e seus respectivos colaboradores abordaram a SFA como uma ferramenta de melhoria nas condições de trabalho, amplamente aceita.

Apresentadas as congruências das variáveis da dimensão tecnológica do CRM, passa-se a apresentar aquelas de cunho organizacional, sintetizadas no QUADRO 2. Perante a definição de planejamento e monitoramento do projeto, a congruência não é total em virtude de apenas um ponto não alinhado na relação entre empresas. A origem pode ser advinda das diferentes linhas e perspectivas de gestão. A Rede Globo atua através de um processo de condução dinâmico perante os negócios. Por outro lado, a HP atua mediante a rigidez de um sistema pré-definido de gestão, o qual pode permitir a existência de gaps, em virtude de uma eventual falta de flexibilidade. O que não permite que esta variável seja considerada congruente na totalidade é a diferença de foco entre empresas. A Rede Globo focaliza sua estratégia empresarial, já a HP o desenvolvimento do software. 
QUADRO 2 - Resultados da Dimensão Organizacional do CRM

\begin{tabular}{|c|c|}
\hline $\begin{array}{l}\text { VARIÁVEIS DA DIMENSÃO } \\
\text { ORGANIZACIONAL DO CRM }\end{array}$ & CONGRUÊNCIAS OBTIDAS NO ESTUDO \\
\hline $\begin{array}{l}\text { Planejamento e } \\
\text { Monitoramento do Projeto de } \\
\text { CRM }\end{array}$ & $\begin{array}{l}\text { O indicador é congruente em praticamente todos os } \\
\text { aspectos, exceto por alguns gaps e pela diferença } \\
\text { de foco das empresas. Foco da HP: Software; foco } \\
\text { da Globo: Estratégia Comercial }\end{array}$ \\
\hline Recursos Humanos & $\begin{array}{l}\text { Na fase atual da parceria HP-Globo existe } \\
\text { congruência, o que no princípio não havia. HP e } \\
\text { Globo interagem mediante um amplo e aberto canal } \\
\text { de comunicação }\end{array}$ \\
\hline $\begin{array}{l}\text { Mudança na Filosofia } \\
\text { Empresarial }\end{array}$ & Indicador congruente na relação HP-Globo-Teoria \\
\hline Poder & $\begin{array}{l}\text { A variável (Poder) apresenta congruência relativa. } \\
\text { As empresas atuam através de diferentes estruturas } \\
\text { de liderança. A HP é rígida e matricial e a Rede } \\
\text { Globo é dinâmica }\end{array}$ \\
\hline
\end{tabular}

Fonte: Elaborado pelos autores.

Quanto aos recursos humanos, existe compreensão da relação empresarial exercida. Na atual fase do projeto, esta é uma variável congruente, o que não pode ser considerado durante a fase inicial da parceria. Os recursos humanos mais relevantes nesta situação são aqueles presentes nos pontos de contato e na tomada de decisão dos atributos e possibilidades do sistema. Treinamento é um aspecto que requer mais atenção, em especial por parte da empresa desenvolvedora da solução. Este pode aumentar a percepção de qualidade e diferenciação perante a empresa cliente usuária, além de maximizar a utilização da ferramenta desenvolvida. A HP pode utilizar-se do fornecimento deste como um diferencial.

Com relação às mudanças na filosofia empresarial, perante o suporte bibliográfico e atitude das organizações, chegou-se a conclusão de que HP e Rede Globo são empresas que exercem mudanças. A própria natureza do negócio entre estas empresas remete à mudança como um processo constante e fundamental. Tanto para a adequação tecnológica, quanto para a adoção e evolução das práticas de CRM, que são efetivadas pela empresa cliente e usuária das soluções, a relação de congruência verificada e necessária é tridimensional. De fato, as empresas HP e TV Globo estão enquadradas no que a teoria referencia, por isso, congruentes.

Perante aspectos de poder, existem congruências relativas, em especial quando da comparação direta entre as empresas. Quanto aos aspectos de poder, os mais interessantes diagnosticados são aqueles incongruentes. As formas de liderança e de gestão são completamente diversas de uma empresa para a outra. A HP atua e é dirigida através de uma estrutura rígida, já a Rede Globo de Televisão é comandada de uma maneira mais dinâmica, e bem menos estruturada. Enquanto que a HP atua mediante a hierarquia rígida, a TV Globo atua mediante a filosofia de 
times de trabalho, de equipes; o que proporciona uma estrutura mais propensa para a delegação de poder, ou empowerment. $O$ aspecto congruente é verificado em razão da existência de uma adequada e bem dimensionada relação de interação entre as empresas, o que ocorre apesar das diferentes modalidades de gestão. Iyer (2002) menciona que as relações iniciais da parceria, e as suas condições e motivações, mudam, tornando pertinente a reavaliação e o reajuste das prioridades. Trata-se de um processo dinâmico, em que a interação determina o grau de sucesso e de maturidade na parceria, e também se esta poderá alcançar os mais altos níveis de colaboração e cooperação.

Os aspectos tecnológicos e os aspectos organizacionais são estruturas de aplicações paralelas, que requerem a atenção de ambas as empresas, tanto da desenvolvedora das soluções quanto da empresa usuária. Para a adequada operação empresarial, e para a manutenção e obtenção de novos clientes, são definidas pelo negócio organizacional as premissas que irão delimitar a atuação perante os clientes, mas que só podem ser colocadas em prática através da utilização das tecnologias adequadas. O alinhamento da tecnologia, tanto de equipamento quanto de software, deve estar adequado com relação à estratégia da organização que a utiliza, e com as pessoas. Deve-se levar em conta a inter-relação entre estrutura, tarefas, tecnologia e pessoas, sendo que a mudança em um fator altera os outros (WILSON, 2000).

\section{Considerações finais}

A relação entre tecnologia, disponibilidade de informação qualificada e organização para a execução das práticas de CRM é praticamente simbiótica e dependente. Existe a necessidade de um alinhamento de ação entre os aspectos citados para que a empresa possa dar uma resposta que implique em obtenção de vantagem competitiva. Embora os aspectos financeiros interfiram na capacidade que a empresa tem de operar, não se trata do único fator relevante com relação à operação do sistema. Existindo os recursos para uma aplicação de CRM, tem-se como elemento crucial a integração da tecnologia pertinente ao padrão adequado de estrutura organizacional, utilizando os conhecimentos sobre o mercado nas decisões da empresa.

Neste sentido, ressalta-se que a gestão da informação deve ser tratada como recurso fundamental ao desempenho da empresa. As fontes de informação que a empresa possui podem ser utilizadas a auxiliar no processo de tomada de decisão (CHOO, 2003). Deve-se fomentar uma cultura organizacional voltada para a busca e para o uso efetivo da informação, que sirva como embasamento ao processo decisório (MAFRA PEREIRA e BARBOSA, 2008) e alimente as ações de gestão de relacionamento com o cliente (CROTEAU e LI, 2003).

Ainda que muitos avanços tenham sido feitos com relação ao melhor atendimento aos clientes, o CRM continua sendo uma prática empresarial sem o devido entendimento (WINER, 2001b). O relacionamento com os 
clientes, embora fundamental, ainda emerge como uma área passível de modificações e de evolução. Muitas vezes, na ânsia de se buscar informações para a gestão do relacionamento, investimentos desnecessários são realizados. Segundo Jamil e Neves (2000), muitas empresas preocuparam-se com a solução emergencial de informação, investindo em computadores, ao invés de projetarem soluções estratégicas; o que resulta em acervos de informações desconexos e desagregados, sem auxílio à tomada de decisão. Neste sentido, Saracevic (1996) aponta para a importância crítica de equacionar a relação homemtecnologia, sugerindo que a aplicação da tecnologia sem objetivos claros pode gerar uma resistência por parte de usuário, sendo necessária a adaptação da tecnologia ao entendimento racional do comportamento humano frente à informação. Saracevic (1996) sugere que as empresas questionem até que ponto as aplicações tecnológicas permitem o eficiente acesso à informação e à comunicação dos estoques disponíveis de conhecimento. Geralmente, segundo o autor, esses pontos ficam esquecidos em meio à sedução das aplicações tecnológicas. $\mathrm{Na}$ investigação da qualidade da informação, inclusive analisando o desperdício de investimento causado pelo uso inadequado da informação, o método infomapping proposto por Burk Jr. e Horton Jr. (1988) pode auxiliar as empresas avaliarem o investimento e o retorno da informação.

Neste estudo, buscou-se investigar a congruência entre os preceitos teóricos, uma empresa desenvolvedora de CRM (HP) e um cliente usuário do sistema (Rede Globo), na utilização de variáveis de gestão de relacionamento sob a ótica tecnológica e organizacional. Todas as variáveis das dimensões propostas se mostraram pertinentes ao estudo desenvolvido, exceto a referente aos sistemas de call center ou CICs, que inexistem na interação entre HP e Rede Globo. Existe uma forte tendência indicando que empresas de tecnologia de ponta utilizam-se das ferramentas diferenciadas de comunicação, fugindo dos padrões tradicionais adotados em geral. Para os contatos diretos com a empresa cliente, a HP utiliza telefones normais, distribuídos aos líderes de setores. Os custos com ferramentas mais elaboradas de comunicação, como os CICs, não são necessários ao tipo de contato da HP com muitos de seus clientes.

Os resultados apresentados, do ponto de vista teórico, podem ser utilizados para uma melhor compreensão dos elementos que compõem as ferramentas e os princípios do CRM. Com base nas dimensões e variáveis constituídas, é possível delimitar e avaliar cada variável de cada dimensão do CRM, acompanhando as modificações e seus respectivos impactos.

Também foi possível investigar no estudo os diferentes campos teóricos e as disciplinas envolvidas, quando se trata de selecionar e utilizar informações de mercado para desenvolver a gestão de relacionamento com clientes. Considerando-se que a informação permeia distintos campos do conhecimento humano, e que para atingir a meta de atender aos problemas da comunicação no contexto empresarial, recuperar e utilizar a informação utiliza-se dos recursos de distintas 
disciplinas, pode-se identificar o caráter interdisciplinar (SARACEVIC, 1996). Como postula Le Coadic (2003), a informação envolve um conteúdo interdisciplinar - ciência da informação, administração, tecnologia da informação, marketing - que deve estar integrado para obter informações relevantes do mercado e dos clientes. Portanto, o CRM também deverá considerar esta abordagem multidisciplinar em seus preceitos.

Outro ponto interessante, observado em especial na entrevista com a Rede Globo (firma usuária do CRM), refere-se à construção de sentido e de significado que a informação sobre clientes e mercado propicia à empresa. É relativamente fácil recuperar dados de clientes ou de outros agentes dentro de uma organização. Entretanto, questões de interpretação e de uso das informações são desafios que as empresas necessitam enfrentar, uma vez que o significado destas informações é determinado nos contextos social e cultural (CAPURRO e HJORLAND, 2007). Ao buscar e usar a informação, as pessoas e as empresas estão continuamente construindo sentido em relação ao ambiente ( $\mathrm{CHOO}$, 2003).

Por fim, no que se refere a implicações gerenciais, as ações de CRM destacam que o monitoramento do ambiente e o conhecimento individualizado dos clientes são elementos fundamentais para 0 estabelecimento de relacionamento de longo prazo com os clientes, em especial daqueles de maior valor, os quais a organização deseja reter e com os quais deseja realizar transações contínuas. São esses clientes os detentores dos recursos desejados pela organização, são desses que a empresa necessita de informação qualificada para incrementar sua gestão de relacionamento.

\section{Referências}

ANGELO, C. F.; GIANGRANDE, V. Marketing de relacionamento no varejo. São Paulo: Atlas, 1999.

BARDIN, L. Análise de Conteúdo. Lisboa: Edições 70, 1977.

BERRY, L. L. Relationship marketing of Services: perspectives from 1983 and 2000. Journal of Relationship Marketing, v.01, n. 01, p.59-77, 2002.

BRETZKE, M. Marketing de relacionamento e competição. São Paulo: Atlas, 2000.

BROWN, S. A. CRM - Customer Relationship Management: uma ferramenta estratégica para o mundo do e-business. São Paulo: Makron Books, 2001.

BRYMAN, A. Research methods and organizational studies. London: Routledge, 1992.

BURK JR., C. F.; HORTON JR., F. W. Infomap: a complete guide to discovering corporate information resources. New York: Englewood Cliffs/Prentice Hall, 1988. 
CAMPBELL, A. J. Creating customer knowledge competence: managing $\mathrm{crm}$ programs strategically. Industrial Marketing Management, v.32, p.375-383, 2003.

CAPURRO, R.; HJORLAND, B. O conceito de informação. Perspectivas em Ciência da Informação, Belo Horizonte, v.12, n.1, 2007.

CERETTA, P. S.; QUADROS, C. J. Implementação do balanced scorecard: um caso prático. REAd - Revista Eletrônica de Administração, n. 34, v.09, p.1-18, 2003.

CHOO, C. W. A organização do conhecimento. São Paulo: SENAC, 2003.

CROTEAU, A.; LI, P. Critical success factors of CRM technological initiatives. Canadian Journal of Administrative Sciences, v.20, n. 01, p.2134, 2003.

DERVIN, B.; MILAN, M. Information needs and uses. Annual Review of Information Science and Technology - ARIST, v.21, 1986.

DOWLING, G. Customer relationship management: In B2C markets, often less is more. California Management Review, v.44, n. 03, p.87-104, 2002.

DRUCKER, P. Sociedade pós-capitalista. São Paulo: Pioneira, 1999.

DWYER, F. R.; SCHURR, P. H.; OH, Sejo. Developing buyer-seller relationships. Journal of Marketing, v.51, p.11-27, 1987.

FERREIRA, S.; SGANZERLLA, S. Conquistando o consumidor: o marketing de relacionamento como vantagem competitiva das empresas. São Paulo: Gente, 2000.

FLETCHER, K. Consumer power and privacy: the changing nature of CRM. International Journal of Advertising, v.22, p.249-272, 2003.

FREITAS, H.; CUNHA Jr., M.V.M.; MOSCAROLA, J. Pelo resgate de alguns princípios da análise de conteúdo: aplicação prática qualitativa em marketing. In: ENCONTRO DA ASSOCIAÇÃO NACIONAL DE PÓSGRADUAÇÃO E PESQUISA EM ADMINISTRAÇÃO - EnANPAD, 20., 1996, Angra dos Reis - RJ. Anais... Rio de Janeiro: ANPAD, 1996. p.467-487.

GIL, A. C . Métodos e técnicas de pesquisa social. 5a Ed. São Paulo: Atlas, 1999. 206p.

GOULART, M. S. B. Uso da informação empresarial no processo de decisão estratégica em empresas de base tecnológica - EBTS: o caso do Centro Empresarial para Laboração de Tecnologias Avançadas - CELTA. Perspectivas em Ciência da Informação, Belo Horizonte, v.12, n.1, 2007.

GRABNER-KRAEUTER, S.; MOEDRITSCHER, G. Alternative approaches toward measuring CRM performance. In: RESEARCH CONFERENCE ON RELATIONSHIP MARKETING AND CUSTOMER RELATIONSHIP MANAGEMENT, 6., 2002, Atlanta. June 9-12, 2002, p.1-16.

GREENBERG, P. CRM at the speed of light: capturing and keeping customers in Internet real time. New York: McGraw Hill, 2002. 
HANSOTIA, B. Gearing up for CRM: antecedents to successful implementation. Journal of Database Marketing, v.10, n. 02, p.121-132, 2002.

HOPEN, N.; LAPOINTE, L. MOREAU, E. Um guia para avaliação de artigos de pesquisas em sistemas de informações. READ - Revista Eletrônica de Administração, v.2, n.2, 1996.

HUGHES, A. M. Data base marketing estratégico. São Paulo: Makron Books, 1998.

Iyer (2002)

JAMIL, G. L.; NEVES, J. T. R. A era da informação: considerações sobre o desenvolvimento das tecnologias da informação. Perspectivas em Ciência da Informação, Belo Horizonte, v.5, n.1, 2000.

LE COADIC, Y-F. A Ciência da Informação. Brasília: Briquet de Lemos, 2003.

LIN, Y.; SU, H-Y. Strategic analysis of customer relationship management: a field study on Hotel Enterprises. TQM \& Business Excellence, v.14, n. 06, p.715-731, 2003.

MAFRA PEREIRA, F. C.; BARBOSA, R. R. Uso de fontes de informação por consultores empresariais: um estudo junto ao mercado de consultoria de Belo Horizonte. Perspectivas em Ciência da Informação, Belo Horizonte, v.13, n.1, 2008.

McKIM, B. The differences between CRM and database marketing. Journal of Database Marketing, v.09, n. 04, p.371-375, 2002.

MIRANDA, S. Como as necessidades de informação podem se relacionar com as competências informacionais. Ciência da Informação, Brasília, v.35, n.3, 2006.

MISSI, F.; ALSHAWI, S.; IRANI, Z. The way forward to a successful customer relationship management. Informing Science, p.1605-1612, 2003.

NEWELL, F. Loyalty.com: customer relationship management in the new era of Internet marketing. New York: McGraw Hill, 2000.

NOGUEIRA, R.; MAZZON, J. A.; TERRA, A. M. A gestão de CRM nas seguradoras. In: ENCONTRO DA ASSOCIAÇÃO NACIONAL DE PÓSGRADUAÇÃO E PESQUISA EM ADMINISTRAÇÃO - EnANPAD, 28., 2004, Curitiba. Anais... Curitiba: ANPAD, 2004.

NORRIS, G.; HURLEY, J. e-Business e ERP: transformando as organizações. Rio de Janeiro: Qualitymark, 2001.

PAAS, L.; KUIJLEN, T. Towards a general definition of customer relationship management. Journal of Database Marketing, v.09, n. 01, p.51-60, 2001. 
PARVATIYAR, A.; SHETH, J. N. Customer relationship management: emerging practice, process, and discipline. Journal of Economic and Social Research, v.03, n. 02, 2001.

PATTON, M.Q. Qualitative evaluation methods. Beverly Hills, CA: Sage, 1986. 381p.

PEDRON, C. D. Estratégia de CRM: Em direção a identificação de indicadores de desempenho. Dissertação (Mestrado em Administração) UNISINOS, 2003.

RAGINS, E. J.; GRECO, A. J. Customer relationship management and ebusiness: more than a software solution. Review of Business, p.25-30, 2003.

ROWE, W. G.; BARNES, J. G. Relationship marketing and sustained competitive advantage. Journal of Market Focused Management, v.02, p.281-297, 1998.

SALAZAR, R. L. A. Padrões de procedimentos para a implantação de um CRM: caso Jet Oil Distribuidora de Produtos de Petróleo Ipiranga. 2002. Dissertação (Mestrado em Administração e Negócios) - Pontifícia Universidade Católica do Rio Grande do Sul - PUCRS, 2002.

SARACEVIC, T. Ciência da informação: origem, evolução e relações. Perspectivas em Ciência da Informação, Belo Horizonte, v.1, n.1, 1996.

SHETH, J. N.; PARVATIYAR, A. Evolving relationship marketing into a discipline. Journal of Relationship Marketing, 2002.

SHOEMAKER, M. E. A framework for examining IT-enabled market relationships. Journal of Personal Selling \& Sales Management, v.21, n. 02, p.177-185, 2001.

SOUZA, M. Os líderes e a busca pelo alto desempenho. Disponível em: $<$ http://www.rh.com.br/ler.php?cod=4103\&org=9 >. Acesso em: 25 de maio 2005.

SPEIER, C.; VENKATESH, V. The hidden minefields in the adoption of sales force automation technologies. Journal of Marketing, v.66, p.98-111, 2002.

SRIVASTAVA, J.; WANG, J-H.; LIM, E-P.; HWANG, S-Y. A case for analytical customer relationship management. Springer-Verlag Berlin Heidelberg, PAKDD 2002, LNAI 2336, p.14-27, 2002.

SWIFT, R. CRM - Customer Relationship Management: o revolucionário marketing de relacionamento com o cliente. Rio de Janeiro: Campus, 2001.

VALENTIM, M. L. P. et al. Gestão da informação utilizando o infomapping. Perspectivas em Ciência da Informação, Belo Horizonte, v.13, n.1, 2008.

WILSON, T. D. Recent trends in user studies and qualitative methods. Information Research, v.5, n. 3, 2000. 
WILSON, H.; DANIEL, E.; MCDONALD, M. Factors for success in customer relationship management (CRM) systems. Journal of Marketing Management, v.18, p.193-219, 2002.

WINER, R. S. A framework for customer relationship management. California Management Review, v.43, n. 04, p.89-105, 2001 a. - Customer relationship management: a framework, research directions, and the future. Haas School of Business, University of California at Berkeley, 2001b.

YIN, R. K. Estudo de caso: planejamento e métodos. 3 ed. Porto Alegre: Bookman, 2005. 